\title{
A comparison of follow-up rates of women with gestational diabetes before and after the updated National Institute for Health and Care Excellence guidance advocating routine follow-up, and the association with neighbourhood deprivation
}

\author{
SEBASTIAN WALSH, ${ }^{1}$ MAHMOUD MAHMOUD,${ }^{2}$ HTWE HTUN, ${ }^{3}$ SHEENA HODGETT, ${ }^{4}$ DAVID BARTON ${ }^{5}$
}

\begin{abstract}
Background: Gestational diabetes mellitus (GDM) occurs in one in every 23 UK pregnancies. GDM identifies the mother as high-risk for development of type 2 diabetes. The National Institute for Health and Care Excellence (NICE) published updated guidance in February 2015 recommending routine follow-up of women with GDM.

Aims: This cohort study compared follow-up rates of women with GDM before and after the updated guidance. We also investigated for an association between follow-up rates and deprivation.

Methods: Participants were identified from the database of the GDM service of two English hospitals and were organised into two cohorts: 'pre-guidance' (2012-2015) and 'post-guidance' (2015-2016). Using the recommendations of the NICE guidance as the follow-up standard, we used the hospitals' computer system to compare follow-up rates of the two cohorts. The English Indices of Deprivation split the country into 32,844 small areas and rank them in order of deprivation such that 1 is the most deprived area and 32,844 is the least deprived. We compared the patients' postcodes against the
\end{abstract}

Specialty Registrar in Public Health Medicine, East of England, UK Foundation Year Two Trainee, Northern Lincolnshire and Goole NHS Foundation Trust, UK

Specialty Registrar in Diabetes and Endocrinology, Shrewsbury and Telford Hospitals NHS Trust, UK

${ }^{4}$ Consultant in Obstetrics and Gynaecology, Shrewsbury and Telford Hospitals NHS Trust, UK

Consultant in Diabetes and Endocrinology, Shrewsbury and Telford Hospitals NHS Trust, UK

Address for correspondence: Dr Sebastian Walsh Endocrinology Secretaries, Princess Royal Hospital Telford, Apley Castle, Apley, Shropshire TF1 6TF, UK

E-mail: Sebastian.walsh@nhs.net

https://doi.org/10.15277/bjd.2019.202
English Indices of Deprivation to investigate the relative levels of neighbourhood deprivation of those followed up compared with those not followed up. The $Z$ statistic was used to test for statistical significance.

Results: 535 participants were included (pre-guidance $n=306$, post-guidance $n=229$ ). Baseline average age (preguidance 32.2 years, post-guidance 32.5 years), body mass index $\left(30.7 \mathrm{~kg} / \mathrm{m}^{2}, 30.9 \mathrm{~kg} / \mathrm{m}^{2}\right)$ and fasting glucose ( $4.9 \mathrm{mmol} / \mathrm{L}, 4.8 \mathrm{mmol} / \mathrm{L})$ were all comparable between cohorts. The follow-up rate improved from $60.5 \%$ in the pre-guidance group to $69.9 \%$ in the post-guidance group. The median deprivation rank of those followed up was 14,565 compared with 13,393 in those not followed up. This difference was not found to be significant.

Conclusion: A higher proportion of women with GDM were followed up with screening for type 2 diabetes after the updated NICE guidance in 2015 recommended routine follow-up. Across the study, over a third of women were not followed up. There was no statistically significant difference in the deprivation levels of those women followed up compared with those not followed up.

Br J Diabetes 2019;19:14-18

Key words: diabetes, gestational, cohort studies, health services research, follow-up studies, health equity

Introduction

Gestational diabetes mellitus (GDM) occurs when women not previously known to have diabetes develop hyperglycaemia during pregnancy. The prevalence of GDM in the UK is estimated to be one in every 23 pregnancies. ${ }^{1}$ Studies suggest that women diagnosed with GDM have a $41 \%$ risk of having GDM again in subsequent pregnancies, ${ }^{2}$ and up to a $60 \%$ lifetime risk of developing type 2 diabetes. ${ }^{3}$ The presence of GDM increases the risk of fetal macrosomia (and the associated obstetric risks of shoulder dystocia and maternal morbidity from caesarean 
section); however, this risk is reduced by optimisation of glycaemic control. ${ }^{4,5}$ Advantages of routine follow-up of women with GDM include the targeting of ongoing risk factors for the development of type 2 diabetes and the opportunity to optimise risk factors before any future pregnancies.

The National Institute of Health and Care Excellence (NICE) published guidance in February 2015 entitled 'Diabetes in pregnancy: management from preconception to the postnatal period'. ${ }^{1}$ It advised that women diagnosed with GDM, whose blood glucose levels returned to normal after delivery, should be followed up with either a fasting blood glucose (FBG) test after 6 weeks or by measuring glycosylated haemoglobin levels $\left(\mathrm{HbA}_{1 \mathrm{c}}\right)$ after 12 weeks. Despite the prevalence and implications of GDM, no nationalised standard for how to follow up women diagnosed with GDM existed prior to the revised guidance in 2015, and follow-up was carried out according to local policies.

The primary research question of this study was: did the rate of follow-up of women diagnosed with GDM change after the updated NICE guidance in 2015? The secondary research question was: was the rate of follow-up associated with deprivation?

\section{Methods}

This was a cohort study using data collected from two district general hospitals in England. Shrewsbury and Telford Hospital NHS Trust (SaTH) operates across two distinct hospital sites: The Royal Shrewsbury Hospital and The Princess Royal Hospital Telford, but both hospitals are administered by one NHS trust and served by a solitary staff body. The trust is the main provider of district general hospital services for nearly half a million people in Shropshire, Telford \& Wrekin and mid Wales. ${ }^{6}$ The population served is predominantly rural with between $90 \%$ and $98 \%$ from the 'white' ethnic group. ${ }^{7}$ The GDM service at SaTH has routinely collected data on women registered with the service since 2012.

There has been a GDM policy in place at SaTH since 2010. The policy was initially based upon the 2008 NICE guidance, and then updated accordingly after the publication of the 2015 guidance. As per the NICE guidance, diagnosis was made based on the results of an oral glucose tolerance test (OGTT) with a FBG level of $\geq 5.6 \mathrm{mmo} / \mathrm{L}(\geq 7.0 \mathrm{mmol} / \mathrm{L}$ before the updated guidance) or 2 -hour plasma glucose level of $\geq 7.8 \mathrm{mmol} / \mathrm{L}$. The 2008 guidance did not contain recommendations for follow-up, however, the SaTH policy was to follow up patients treated with dietary advice only with a 6-week postnatal FBG test requested from the GP in the discharge paperwork, and those treated with oral agents or insulin with an OGTT organised by the GDM service itself. There was no change to the SaTH follow-up policy after the publication of the 2015 guidance.

We combined data collected by the GDM service (demographics, reason for having the diagnostic OGTT and treatment for GDM) with data from the hospital computer system (delivery date, results and timing of any follow-up tests performed).

Women were included if they had been registered with the GDM service during or after 2012 until the end of 2016 (this was to allow for the follow-up period to have elapsed). Participants were excluded if the dataset was not sufficiently complete to be confident of whether or not follow-up had occurred (eg, hospital number not recorded, delivery date not identifiable from computer system).

Participants were split into two cohorts, those who delivered on or after 1 January 2012 until before the updated NICE guidance was implemented at SaTH on 1 April 2015 and those who gave birth on or after this date until 31 December 2016. Those who delivered before implementation of the NICE guidance were identified as the 'pre-guidance' cohort and those who delivered after the guidance was implemented were identified as the 'post-guidance' cohort.

The outcome measure was the success or failure of followup of women according to the 2015 NICE guidance. Participants were deemed to have been followed up appropriately if they had either a FBG test performed 6-13 weeks post-delivery or a $\mathrm{HbA}_{1 \mathrm{c}}$ blood test performed 13-24 weeks post-delivery. NICE guidance does not actually specify a maximum timeframe for follow-up to occur, merely that it should. Maximum timeframes used in other studies ranged from 12 weeks to several years.8,9 We used 24 weeks as the cut-off as a balance between allowing sufficient time for follow-up to occur against the pragmatism of needing the follow-up time period to have elapsed before performing the study.

The English Indices of Deprivation are a measure of relative deprivation in small areas in England called lower-layer super output areas (LSOA) published by the UK government. ${ }^{10}$ The indices are a composite measure calculated by collating data on income, employment, education, health and disability, crime, barriers to housing and services, housing quality and the environment. There are 32,844 LSOAs in total and they are ranked such that ' 1 ' is the most deprived area and the area ranked ' 32,844 ' is the least deprived. By inputting the included women's postcodes into the interactive web tool (published alongside the index), we were able to compare the average index rank of women who were followed up successfully with those who were lost to follow-up, and thus create a proxy correlation of followup rates with deprivation. A statistical comparison of the datasets was undertaken on Microsoft Excel using the Z test.

\section{Results}

\section{Baseline characteristics}

The baseline characteristics of the two cohorts are shown in Table 1. Reasons for screening were also similar between cohorts, with the most common reasons for performing an OGTT being: first degree relative with diabetes $(n=109, n=75)$, body mass index $>30 \mathrm{~kg} / \mathrm{m}^{2}(\mathrm{n}=84, \mathrm{n}=62)$, and ultrasound estimate of fetal growth above the $90^{\text {th }}$ customised centile for gestation ( $n=54, n=50)$.

In the pre-guidance cohort, $9.2 \%$ of participants were treated with metformin as a sole agent, $2.9 \%$ were treated with insulin as a sole agent, and 3.9\% were treated with a combination of both. In comparison, in the post-guidance cohort, more women were treated with insulin (17.5\%) compared to only $4.8 \%$ with metformin and $4.8 \%$ with a combination of both. 
Table 1 Baseline characteristics of the pre- and post-guidance cohorts

\begin{tabular}{lll}
\hline & $\begin{array}{l}\text { Pre-guidance } \\
\text { cohort }\end{array}$ & $\begin{array}{l}\text { Post-guidance } \\
\text { cohort }\end{array}$ \\
\hline Number of women & 306 & 229 \\
Mean age (years) & 32.2 & 32.5 \\
Mean BMI (kg/m²) & 30.7 & 30.9 \\
Mean fasting glucose (mmol/L) & 4.9 & 4.8 \\
\% treated with metformin only & 9.2 & 4.8 \\
\% treated with insulin only & 2.9 & 17.5 \\
\% treated with combination & 3.9 & 4.8 \\
\% treated with any drug & 16.0 & 27.1 \\
\hline
\end{tabular}

However, the average FBG reading at diagnosis did not differ significantly between the cohorts $(4.9 \mathrm{mmol} / \mathrm{L}$ and $4.8 \mathrm{mmol} / \mathrm{L}$, respectively), so it is likely that this discrepancy in treatment frequencies represents shifting therapeutic approaches to treating GDM rather than any intrinsic differences between the cohorts.

We excluded participants from the analysis if we were unable to elicit the delivery date from the computer system. This occurred in only nine cases in the pre-guidance cohort and seven cases in the post-guidance cohort.

\section{Outcome}

In the pre-guidance cohort, $57.5 \%$ of women were followed up by use of a FBG test and $6.5 \%$ were followed up by $\mathrm{HbA}_{1 \mathrm{c}}$. Accounting for women who were followed up with both methods, a total of $60.5 \%$ of the pre-guidance cohort were followed up successfully. In contrast, in the post-guidance cohort $62.0 \%$ of women were followed up with a FBG test and $17.5 \%$ with $\mathrm{HbA}_{1 \mathrm{c}}$, giving a total of $69.9 \%$ of the cohort successfully followed up.

A total of $2.8 \%$ of the women followed up were found to have $\mathrm{FBG}$ readings or $\mathrm{HbA}_{1 \mathrm{c}}$ results in the diabetic range as stipulated by NICE (FBG $\geq 7 \mathrm{mmol} / \mathrm{L}, \mathrm{HbA}{ }_{1 \mathrm{c}} \geq 48 \mathrm{mmol} / \mathrm{mol}(6.5 \%)$ ). In the pre-guidance cohort the rate of diabetes at follow-up was $2.6 \%$ compared with $3.1 \%$ in the post-guidance cohort.

The rate of follow-up was not strongly associated with baseline FBG $(60.7 \%$ follow-up rate amongst women with baseline FBG $\geq 5.6 \mathrm{mmol} / \mathrm{L}$ compared with $57.4 \%$ follow-up rate amongst women with baseline FBG $<5.6 \mathrm{mmol} / \mathrm{L}$ ). There was, however, a strong association between type of GDM treatment and the incidence of follow-up: $80.2 \%$ of women treated with either metformin or insulin were followed up compared with only $60.1 \%$ of women who were treated with dietary advice alone.

\section{Deprivation}

The median LSOA deprivation rank of those women followed up successfully was 14,565 compared with 13,393 for those women not followed up successfully. Forty-six participants had to be excluded from this analysis because they lived in Wales and the Index is produced separately for England and Wales. We tested for statistical significance using the $Z$ test, with a threshold of $p<0.05$ to reject the null hypothesis of no significant difference between the two datasets. We found a $p$ value of 0.16 , which meant there was insufficient evidence to reject the null hypothesis. We therefore conclude that the observed association between deprivation and being lost to follow-up was not statistically significant.

\section{Discussion}

\section{Main findings}

In this study of follow-up rates among 535 women with GDM at two district general hospitals in England, the rate of follow-up improved from $60.5 \%$ to $69.9 \%$ after the publication of updated NICE guidance. There was no statistically significant relationship between neighbourhood deprivation and follow-up rates.

\section{Strengths and limitations}

There are several strengths of this study. We included a large number of participants and, to the best of our knowledge, they represented the entire GDM population of the trust during the study period. This, coupled with the very small number of participants with missing data, limits the potential for bias.

The participants included in this study were sourced exclusively from the records of the GDM team at SaTH, who aim to record data from all women registered with the service. We were not able to externally confirm that this represented the entire population and it is possible that there are women who were missed from registration on the database - for example, because of administrative errors. However, this is likely to represent a very small number of participants, if any.

The main limitation of this study was that it was performed using data from only one NHS trust. Although women from two hospitals were included, a single GDM multidisciplinary team serves both hospitals, limiting the generalisability of the findings.

\section{Interpretation of findings in relation to previously published work}

To the best of our knowledge, this is the first study to investigate how the follow-up rate of women with GDM has changed following the updated NICE guidance. This, coupled with the limited generalisability of the study setting, limits the external validity of the results.

This is an observational study and can therefore not be used to infer a causal process, rather than to observe a correlation. We must therefore consider the possibility of confounding factors. It is possible that another factor, other than the publication of NICE guidance, was responsible for the observed improvement in the follow-up rates. For example, it is possible that the mere collection of participants' details in the database by the GDM team (commenced in 2012) leads itself to more vigilance around follow-up. Indeed, the follow-up rate of $>60 \%$ is generally higher than that reported by previous studies. $8,11-15$ However, 
it should be noted that the team did not actually collect data on who had and had not been followed up.

While there was a small improvement in the proportion of women being followed up by FBG level (pre-guidance 57.5\%, post-guidance $62.0 \%$ ), the majority of the improvement in follow-up rates between the pre- and post-guidance cohorts was a large increase in the use of a $\mathrm{HbA}_{1 c}$ test to follow up women, from $6.5 \%$ to $17.5 \%$. This is likely to be partly because of the incorporation of $\mathrm{HbA}_{1 \mathrm{c}}$ in the updated NICE guidance. It is possible that the convenience of the $\mathrm{HbA}_{1 \mathrm{c}}$ test (involving a one-off blood test rather than the relatively time-consuming OGTT) influenced this finding.

Across the study, women who were treated with insulin or metformin rather than only receiving dietary intervention were far more likely to be followed up ( $80.2 \%$ vs. $60.1 \%$ ). This could be because those women had more interactions with healthcare professionals during their pregnancy, because the treatment made them more conscious of their diagnosis, or because follow-up was performed by the GDM team itself. The percentage of women treated with any agent increased by $11.1 \%$ from the pre-guidance to the post-guidance group (from $16.0 \%$ to $27.1 \%$ ), predominantly due to an increased number of women using insulin. It is likely that this also accounts for some of the increase in follow-up observed between the two cohorts. The increase in women being treated with insulin or metformin in the post-guidance group is likely to be because of a change in the target blood glucose levels in the updated NICE guidance (from therapeutic targets of $\leq 6.0 \mathrm{mmol} / \mathrm{L}$ (fasting) and $\leq 8.0$ $\mathrm{mmol} / \mathrm{L}$ ( 2 hour) pre-guidance to post-guidance targets as per NICE 2015 of $\leq 5.3 \mathrm{mmol} / \mathrm{L}$ (fasting), $\leq 7.8 \mathrm{mmol} / \mathrm{L}$ (1 hour) and $\leq 6.4 \mathrm{mmol} / \mathrm{L}$ (2 hour)).

Taking the above caveats into account, we observed a large improvement in the rate of follow-up of women with GDM after the publication of updated NICE guidance that advocated routine follow-up. There is no persuasive argument for why this observation should have occurred by chance, because of bias, or by the action of some external factor. Our interpretation of the results is that it is likely that the publication of updated NICE guidance improved the rate of follow-up of women with GDM in this population.

Another interesting finding in the study was the increased rate of diagnosis of GDM as the study progressed. This was evidenced by an increase from 7.8 diagnoses/month in the preguidance group to 19.1 diagnoses/month in the post-guidance group. As mean age and body mass index were comparable between the two groups, it is likely that this increase in incidence was a result of the change in diagnostic criteria from a FBG level of $\geq 7.0 \mathrm{mmol} / \mathrm{L}$ (pre-guidance) to $\geq 5.6 \mathrm{mmol} / \mathrm{L}$ (post-guidance).

Implications for future research, policy and practice

Although this study showed an improvement in the rate of follow-up of women with GDM during the study period, over one-third of the participants were not followed up. Given the high incidence of type 2 diabetes after a GDM diagnosis, this leaves a large number of high-risk individuals who are not being actively followed up.

\section{Key messages}

- Follow up of women with GDM improved after the publication of updated NICE guidance in 2015

- This may be due to higher rates of treatment with insulin, and/or the incorporation of $\mathrm{HBA}_{1 \mathrm{c}}$ into the follow-up guidance

- Across the study, over a third of women with GDM were not followed up

Evidence from this study suggests that offering follow-up with a $\mathrm{HbA}_{1 \mathrm{c}}$ could provide an opportunity to increase postnatal screening rates and future research could test this hypothesis more directly.

This study was performed in a focused study setting and further research should now be undertaken to investigate whether trends observed in this study are true across a wider range of settings.

\section{Conclusion}

A higher proportion of women diagnosed with GDM were followed up with screening for type 2 diabetes after the publication of updated NICE guidance in 2015 advocating routine follow-up of these women. Possible explanations for the increased followup rate include: the increased proportion of women receiving drug treatment owing to stricter therapeutic targets and the incorporation of $\mathrm{HbA}_{1 \mathrm{c}}$ as a follow-up test in the guidance.

However, over a third of women were not followed up and this potentially represents a large number of women across the country at high risk of type 2 diabetes who are not actively being followed up. In our study we found no statistically significant relationship between neighbourhood deprivation and follow-up rates.

Conflict of interest The authors declare that they have no conflicts of interest in relation to this article.

\section{Funding None.}

Contribution statement The idea for the study was conceived by DB. SW, ME and $\mathrm{HH}$ completed data collection. SW performed the data analysis and drafted the manuscript. DB provided feedback during the conduct of the research. SH and DB commented on the final draft.

Novelty statement To the best of our knowledge, this is the first study which investigates the effect of the publication of updated NICE guidance advocating routine follow-up of women with GDM on follow-up rates in a realworld setting. The study demonstrates that up to one-third of women at high risk of developing type 2 diabetes are not being followed up.

HRA approval Project ID: 247335

\section{References}

1. National Institute for Health and Care Excellence (NICE). Diabetes in pregnancy: management from preconception to the postnatal period. NG3. February 2015. www.nice.org.uk

2. Getahun D, Fassett M, Jacobsen S. Gestational diabetes: risk of recurrence in subsequent pregnancies. Am J Obstet Gynecol 2010;203:467. https://doi.org/10.1016/j.ajog.2010.05.032 
3. Noctor E, Dunne F. Type 2 diabetes after gestational diabetes: the influence of changing diagnostic criteria. World J Diabetes 2015;6:234-44. https://doi.org/10.4239/wjd.v6.i2.234

4. Crowther C, Hiller J, Moss J, McPhee A. Effect of treatment of gestational diabetes mellitus on pregnancy outcomes. N Engl J Med 2005;352:247786. https://doi.org/10.1056/NEJMoa042973

5. Gonzalez-Quintero $V$, Istwan N, Rhea $D$, et al. The impact of glycemic control on neonatal outcome in singleton pregnancies complicated by gestational diabetes. Diabetes Care 2007;30:467-70. https://doi.org/ 10.2337/dc06-1875

6. Shrewsbury and Telford NHS Hospital Trust (SaTH). https://www.sath.nhs.uk/ about-us/

7. Office for National Statistics. 2011 Census. https://www.ons.gov.uk/census/2011census

8. Nielsen K, Kapur A, Damm P, de Courten M, Bygbjerg C. From screening to postpartum follow-up - the determinants and barriers for gestational diabetes mellitus (GDM) services, a systematic review. BMC Pregnancy Childbirth 2014;14:41. https://doi.org/10.1186/1471-2393-14-41.

9. Kjos S, Peters R, Xiang A, Henry O, Montoro M, Buchanan T. Predicting future diabetes in Latino women with gestational diabetes: utility of early postpartum glucose tolerance testing. Diabetes 1995;44:586-91. https://doi.org/10.2337/diab.44.5.586
10. Ministry of Housing, Communities and Local Government. English indices of deprivation 2015. https://www.gov.uk/government/statistics/english-indices-of-deprivation-2015

11. Smirnakis K, Chasan-Taber L, Wolf M, Markenson G, Ecker J, Thadhani R. Postpartum diabetes screening in women with a history of gestational diabetes. Obstet Gynecol 2005;106:1297-303. https://doi.org/10.1097/ 01.AOG.0000189081.46925.90

12. Stasenko M, Cheng Y, McLean T, Jelin A, Rand L, Caughey A. Postpartum follow-up for women with gestational diabetes mellitus. Am J Perinatol 2010;27:737-42. https://doi.org/10.1055/s-0030-1253557

13. Stasenko M, Liddell J, Cheng Y, Sparks T, Killion M, Caughey A. Patient counseling increases postpartum follow-up in women with gestational diabetes mellitus. Am J Obstet Gynecol 2011;204:522.e1-522.e6. https://doi.org/10.1016/j.ajog.2011.01.057

14. Ward R, Hanna F, Shelley-Hitchen A, et al. Gestational diabetes mellitus: are mothers being followed-up? A retrospective study. $\mathrm{Br} / \mathrm{Gen}$ Pract 2018;68(Suppl 1). https://doi.org/10.3399/bjgp18X697469

15. Rosenbloom JI, Blanchard MH. Compliance with postpartum diabetes screening recommendations for patients with gestational diabetes. J Womens Health 2018;27:498-502. https://doi.org/10.1089/jwh.2017.6477

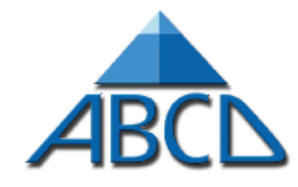

Association of British Clinical Diabetologist:

\section{Semaglutide Nationwide Audit in progress}

\author{
If yes, REGISTER YOUR CENTRE! \\ http://www.diabetologists-abcd.org.uk/GLP1_Audits/Semaglutide_Audit.htm
}

- invited to enter your patient data into the bespoke online tool

- you can collect data on the easy-to-complete paper proformas which you can printout from the above web address

- you are able to analyse your local data easily

- the data will be automatically added to the national data in anonymised form

Please remember:

- the more data, the more complete our understanding of Semaglutide in real clinical practice

- all contributors will be listed in publications arising from data submission 